\title{
Global Stability of Dynamic Systems of High Order
}

\author{
Mohammed BENALILI and Azzedine LANSARI
}

Department of Mathematics, B.P. 119, Faculty of Sciences, University Abou-bekr BelKaïd, Tlemcen, Algeria

E-mail: m_benalili@mail.univ-tlemcen.dz,a_lansari@mail.univ-tlemcen.dz

Received December 18, 2006, in final form June 04, 2007; Published online July 15, 2007

Original article is available at http://www.emis.de/journals/SIGMA/2007/077/

\begin{abstract}
This paper deals with global asymptotic stability of prolongations of flows induced by specific vector fields and their prolongations. The method used is based on various estimates of the flows.
\end{abstract}

Key words: global stability; vector fields; prolongations of flows

2000 Mathematics Subject Classification: 37C10; 34D23

\section{Introduction}

Global stability of dynamic systems is a vast domain in ordinary differential equations and it is one of its main topics. Many works have been done in this context, we list some of them: $[3,4,5,6,7,8]$. However, little is known in the stability of high order (see [10] and [2]). In this paper, we are concerned with the global asymptotic stability of prolongations of flows generated by some specific vector fields and their perturbations. The method used is based on various estimates of the flows and their prolongations. To justify the study of the dynamic of prolongations of flows, we consider the Lie algebra $\chi\left(\mathbb{R}^{n}\right)$ of vector fields on $\mathbb{R}^{n}$ endowed with the weak topology, which is the topology of the uniform convergence of vector fields and all their derivatives on a compact sets. The Lie bracket is a fundamental operation not only in differential geometry but in many fields of mathematics, such as dynamic and control theory. The invertibility of this latter is of many uses i.e. given any vector fields $X, Z$ find a vector field $Y$ such that $[X, Y]=Z$. In the case of vector fields $X$ defined in a neighborhood of a point $a$ with $X(a) \neq 0$ we have a positive answer: since in this case the vector field $X$ is locally of the form $\frac{\partial}{\partial x_{1}}$ and the solution is given by

$$
Y\left(x_{1}, \ldots, x_{n}\right)=\int_{-r}^{x_{1}} Z\left(t, x_{2}, \ldots, x_{n}\right) d t
$$

where $\|x\|=\max _{1 \leq i \leq n}\left|x_{i}\right|<r$. In the case of singular vector fields, i.e. $X(a)=0$ little is known. Consider a singular vector field $X$ defined in a neighborhood $U$ of the origin 0 with $X(0)=0$ and let $\phi_{t}$ be the flow generated by $X$. Suppose that $X$ is complete and consider a vector field $Y$ defined on an open set $V \supset \phi_{t}(U)$ for all $t \in \mathbb{R}$. The transportation of a vector field $Y$ along the flow $\phi_{t}$ is defined as

$$
\left(\phi_{t}\right)_{*} Y(x)=\left(D \phi_{t} \cdot Y\right) \circ \phi_{-t}(x)
$$

and the derivative with respect to $t$ is given as follows

$$
\frac{d}{d t}\left(\phi_{t}\right)_{*} Y=\left[\left(\phi_{t}\right)_{*} X,\left(\phi_{t}\right)_{*} Y\right] .
$$


Put $Y_{t}=-\int_{0}^{t}\left(\phi_{s}\right)_{*} Z d s$, then

$$
\left[X, Y_{t}\right]=-\left.\frac{d}{d t}\right|_{t=0}\left(\phi_{t}\right)_{*} \int_{0}^{t}\left(\phi_{s}\right)_{*} Z d s=-\int_{0}^{t} \frac{d}{d s}\left(\phi_{s}\right)_{*} Z d s=Z-\left(\phi_{t}\right)_{*} Z
$$

So if $\left(\phi_{t}\right)_{*} Z$ converges to 0 and the integral $Y=-\int_{0}^{+\infty}\left(\phi_{s}\right)_{*} Z d s$ is convergent in the weak topology, then $Y$ is a solution of our equation.

As applications of the right invertibility of the bracket operation on germs of vector fields at a singular point we refer the reader to the papers by the authors $[1,2]$ (see also [10]).

\section{Generalities}

First we recall some definitions on global asymptotic stability as introduced in [9]. Let $\|\cdot\|$ be the Euclidean norm on $\mathbb{R}^{n}, K \subset \mathbb{R}^{n}$ is a compact set and $f$ any smooth function on $\mathbb{R}^{n}$, we put

$$
\|f\|_{r}^{K}=\sup _{x \in K} \max _{|\alpha| \leq r}\left\|D^{\alpha} f(x)\right\|
$$

Definition 1. A point $a \in \mathbb{R}^{n}$ is said globally asymptotically stable (in brief G.A.S.) of the flow $\phi_{t}$ if

i) $a$ is an asymptotically stable (in brief A.S.) equilibrium of the flow $\phi_{t}$;

ii) for any compact set $K \subset \mathbb{R}^{n}$ and any $\varepsilon>0$ there exists $T_{K}>0$ such that for any $t \geq T_{K}$ we have $\left\|\phi_{t}(x)-a\right\| \leq \epsilon$ for all $x \in K$.

Definition 2. The point $a \in \mathbb{R}^{n}$ is said globally asymptotically stable of order $r(1 \leq r \leq \infty)$ for the flow $\phi_{t}$ if

i) $a$ is a G.A.S. point for the flow $\phi_{t}$;

ii) for any compact set $K \subset \mathbb{R}^{n}$ and

$$
\forall \epsilon>0, \quad \exists T_{K}>0 \text { such that } \forall t \geq T_{K} \Rightarrow\left\|\phi_{t}-a I\right\|_{r}^{K} \leq \epsilon,
$$

where $I$ denotes the identity map.

A vector field $X$ will be called semi-complete if the $X$-flow $\phi_{t}=\exp (t X)$ is defined for all $t \geq 0$.

First we quote the following proposition which characterizes the uniform asymptotic stability, for a proof see the book of W. Hahn [5].

Let $(\phi)_{t}$ denote a flow defined on $\mathbb{R}^{n}$.

Proposition 1. The origin 0 in $\mathbb{R}^{n}$ is G.A.S. point for the flow $\phi_{t}$ if for any ball $B(0, \rho)$, centered at 0 and of radius $\rho>0$, there exist $t_{0} \geq 0$ and functions $a, b$ such that

$$
\left\|\phi_{t}(x)\right\| \leq a(\|x\|) b(t)
$$

with a a continuous function on $B(0, \rho)$ monotonously increasing such that $a(0)=0$ and $b$ is a continuous function defined for any $t \geq t_{0}$ monotonously decreasing such that $\lim _{t \rightarrow+\infty} b(t)=0$.

\section{Estimates of prolongations of flows}

We start with some perturbations of linear vector fields. 


\subsection{Perturbation of linear vector fields}

Consider the following linear vector field

$$
X_{1}=\sum_{i=1}^{n} \alpha_{i} x_{i} \frac{\partial}{\partial x_{i}}
$$

where the coefficients $\alpha_{i} \in[a, b] \subset \mathbb{R}$ and are not all 0 .

The $X_{1}$-flow, $\psi_{t}^{1}=\exp \left(t X_{1}\right)$ is then

$$
\psi_{t}^{1}(x)=x e^{\alpha t}=\left(x_{1} e^{\alpha_{1} t}, \ldots, x_{n} e^{\alpha_{n} t}\right) \quad \forall t \in \mathbb{R}
$$

and its estimates are given by

$$
\|x\| e^{a t} \leq\left\|\psi_{t}^{1}(x)\right\| \leq\|x\| e^{b t} .
$$

Consider now a perturbation of the vector field $X_{1}$ of the form $Y_{1}=X_{1}+Z_{1}$, where $Z_{1}$ is a smooth vector field globally Lipschitzian on $\mathbb{R}^{n}$. The explicit form of the $Y_{1}$-flow is then

$$
\psi_{t}^{1}(x)=x e^{A t}+\int_{0}^{t} Z_{1}\left(\psi_{s}^{1}(x)\right) d s,
$$

where $A=\left(\begin{array}{ccc}\alpha_{1} & \cdots & 0 \\ \vdots & \ddots & \vdots \\ 0 & \cdots & \alpha_{n}\end{array}\right)$.

Lemma 1. If the perturbation $Z_{1}$ fulfills

$$
\left\|Z_{1}(x)\right\| \leq c_{0} \quad \forall x \in \mathbb{R}^{n}
$$

then the vector field $Y_{1}$ is complete and the $Y_{1}$-flow satisfies the estimates

$$
\left(\|x\|-\frac{c_{0}}{a}\right) e^{b t}+\frac{c_{0}}{a} \leq\left\|\psi_{t}^{1}(x)\right\| \leq\left(\|x\|+\frac{c_{0}}{b}\right) e^{b t}-\frac{c_{0}}{b} .
$$

Proof. Clearly the $Y_{1}$-flow $\psi_{t}^{1}$ is bounded for any $t \in[0, T]$ with $T<+\infty$ and any $x \in \mathbb{R}^{n}$. The same is true if we replace $t$ by $-t$. Then $\psi_{t}^{1}$ is complete.

Consider now the equation

$$
\frac{1}{2} \frac{d}{d t}\left\|\psi_{t}^{1}(x)\right\|^{2}=\left\langle\psi_{t}^{1}(x), \alpha \psi_{t}^{1}(x)+Z_{1}\left(\psi_{t}^{1}(x)\right)\right\rangle .
$$

Letting $y=\left\|\psi_{t}^{1}(x)\right\|$, we deduce

$$
a y^{2}-c_{0} y \leq \frac{1}{2} \frac{d}{d t} y^{2} \leq b y^{2}+c_{0} y, \quad y(0)=\|x\|
$$

and by integrating we obtain

$$
\left(\|x\|-\frac{c_{0}}{a}\right) e^{b t}+\frac{c_{0}}{a} \leq y \leq\left(\|x\|+\frac{c_{0}}{b}\right) e^{b t}-\frac{c_{0}}{b} .
$$

Let $B(0,1)$ be the open unit ball centered at the origin 0 . 
Lemma 2. If the perturbation $Z_{1}$ fulfills the estimates

$$
\begin{aligned}
& \left\|Z_{1}(x)\right\| \leq c_{0}^{\prime}\|x\|^{1+m} \quad \forall x \in B(0,1) \text { and any integer } m \geq 1, \\
& \left\|Z_{1}(x)\right\| \leq c_{0}^{\prime \prime}\|x\| \quad \text { for every } x \in \mathbb{R}^{n} \backslash B(0,1),
\end{aligned}
$$

then $Y_{1}$ is complete and the $Y_{1}$-flow fulfills the following estimates for ant $t \geq 0$

$$
\begin{aligned}
& \|x\| e^{a_{0} t} \leq\left\|\psi_{t}^{1}(x)\right\| \leq\|x\| e^{b_{0} t}, \\
& \|x\| e^{-b_{0} t} \leq\left\|\psi_{-t}^{1}(x)\right\| \leq\|x\| e^{-a_{0} t}
\end{aligned}
$$

with $c_{0}=\max \left\{c_{0}^{\prime}, c_{0}^{\prime \prime}\right\}, a_{0}=a-c_{0}$ and $b_{0}=b+c_{0}$.

Proof. Taking account of the explicit form of the flow (5) and the estimates (8), we deduce that $Y_{1}$ is complete. If $x \in B(0,1)$ then $\left\|Z_{1}(x)\right\| \leq c_{0}^{\prime}\|x\|^{1+m} \leq c_{0}^{\prime}\|x\|$, letting $c_{0}=\max \left\{c_{0}^{\prime}, c_{0}^{\prime \prime}\right\}$ then $\left\|Z_{1}(x)\right\| \leq c_{0}\|x\|$ for any $x \in \mathbb{R}^{n}$. If we put $y=\left\|\psi_{t}^{1}(x)\right\|$ the equation (7) leads to

$$
\left(a-c_{0}\right) y \leq \frac{d}{d t} y \leq\left(b+c_{0}\right) y, \quad y(0)=\|x\|
$$

and putting $b_{0}=b+c_{0}, a_{0}=a-c_{0}$, we deduce the following estimates

$$
\|x\| e^{a_{0} t} \leq y \leq\|x\| e^{b_{0} t} \text { for any } t \geq 0 .
$$

The same is also true in the on $\mathbb{R}^{n} \backslash B(0,1)$.

Lemma 3. Suppose that all the coefficients $\alpha_{i}$ are negative, $a \leq \alpha_{i} \leq b<0$.

If the perturbation $Z_{1}$ fulfills the estimates

$$
\left\|Z_{1}(x)\right\| \leq c_{0}\|x\|^{1+m} \quad \text { for any } x \in \mathbb{R}^{n} \text { and any integer } m \geq 1,
$$

then the vector field $Y_{1}$ is semi-complete and the $Y_{1}$-flow satisfies the estimates for any $t \geq 0$

$$
\begin{aligned}
& \|x\| e^{a t}\left(1-\frac{c_{0}}{a}\|x\|^{m}\left(1-e^{a m t}\right)\right)^{-\frac{1}{m}} \\
& \quad \leq\left\|\psi_{t}^{1}(x)\right\| \leq\|x\| e^{b t}\left(1-\frac{c_{0}}{b}\|x\|^{m}\left(1-e^{b m t}\right)\right)^{-\frac{1}{m}} .
\end{aligned}
$$

Proof. By the relation (5) and the estimates (10), we deduce that the vector field $Y_{1}$ is semicomplete. Letting $y=\left\|\psi_{t}^{1}(x)\right\|$ and taking into account the equation (7) and the estimates (10) we deduce that

$$
a y-c_{0} y^{1+m} \leq \frac{d}{d t} y \leq b y+c_{0} y^{1+m}, \quad y(0)=\|x\|
$$

and by integration we have

$$
\|x\| e^{a t}\left(1-\frac{c_{0}}{a}\|x\|^{m}\left(1-e^{a m t}\right)\right)^{-\frac{1}{m}} \leq y \leq\|x\| e^{b t}\left(1-\frac{c_{0}}{b}\|x\|^{m}\left(1-e^{b m t}\right)\right)^{-\frac{1}{m}} .
$$

Example 1. Let the vector field

$$
X_{3}=\sum_{i=1}^{n}\left(\alpha_{i} x_{i}+\beta_{i} x_{i}^{1+m_{i}}\right) \frac{\partial}{\partial x_{i}}
$$

such that all the coefficients fulfilling

$$
a \leq \alpha_{i} \leq b<0, \quad a^{\prime} \leq \beta_{i} \leq b^{\prime} \leq 0
$$


and all the exponents $m_{i}$ are even positive integers with $0<m_{0}^{\prime} \leq m_{i} \leq m_{0}$. The associated flow $\phi_{t}^{3}=\exp \left(t X_{3}\right)$ is the solution of the dynamic system

$$
\frac{d}{d t} \phi_{t}(x)=X_{3} \circ \phi_{t}(x), \quad \phi_{0}(x)=x
$$

or in coordinates

$$
\frac{d}{d t}\left(\phi_{t}(x)\right)_{i}=\alpha_{i}\left(\phi_{t}(x)\right)_{i}+\beta_{i}\left(\phi_{t}(x)\right)_{i}^{1+m_{i}}, \quad \phi_{0}(x)=x .
$$

This latter is a Bernoulli type equation and its solution is given by

$$
\left(\phi_{t}^{3}(x)\right)_{i}=x_{i} e^{\alpha_{i} t}\left(1+\frac{\beta_{i}}{\alpha_{i}} x_{i}^{m_{i}}\left(1-e^{\alpha_{i} m_{i} t}\right)\right)^{\frac{-1}{m_{i}}} .
$$

The $X_{3}$-flow $\phi_{t}^{3}=\exp \left(t X_{3}\right)$ then has the explicit form

$$
\phi_{t}^{3}(x)=x e^{\alpha t}\left(1+\frac{\beta}{\alpha} x^{m}\left(1-e^{\alpha m t}\right)\right)^{\frac{-1}{m}}
$$

and the following estimates are true, $\forall t \geq 0$

$$
\|x\| e^{a t} \leq\left\|\phi_{t}^{3}(x)\right\| \leq\|x\| e^{b t} .
$$

\subsection{Estimation of the $k^{\text {th }}$ prolongation of the $Y_{1}$-flow}

Denote by $\eta_{1}^{1}(t, x, \nu)=D \psi_{t}^{1}(x) \nu$, where $\nu \in \mathbb{R}^{n}$, the first derivative with respect to $x$ of the $Y_{1}$-flow, solution of the dynamic system

$$
\frac{d}{d t} \eta_{1}^{1}(t, x, \nu)=\left(D_{y} X_{1}+D_{y} Z_{1}\right) \eta_{1}^{1}(t, x, \nu), \quad \eta_{1}^{1}(0, x, \nu)=\nu
$$

with $y=\psi_{t}^{1}(x)$.

Lemma 4. If the perturbation $Z_{1}$ fulfills the estimate

$$
\left\|D Z_{1}(x)\right\| \leq c_{1} \quad \text { for any } x \in \mathbb{R}^{n},
$$

then the derivative of the $Y_{1}$-flow is complete and has the following estimates, for any $t \geq 0$

$$
e^{a_{1} t} \leq\left\|D \psi_{t}^{1}(x)\right\| \leq e^{b_{1} t}, \quad e^{-b_{1} t} \leq\left\|D \psi_{-t}^{1}(x)\right\| \leq e^{-a_{1} t}
$$

with $a_{1}=a-c_{1}$ and $b_{1}=b+c_{1}$.

Proof. Consider as in previous lemmas the following equation

$$
\frac{1}{2} \frac{d}{d t}\left\|\eta_{1}^{1}(t, x, \nu)\right\|^{2}=\left\langle\eta_{1}^{1}(t, x, \nu),\left(\alpha+D Z_{1}\right) \eta_{1}^{1}(t, x, \nu)\right\rangle
$$

and put $z=\left\|\eta_{1}^{1}(t, x, \nu)\right\|$, so

$$
\left(a-c_{1}\right) z^{2} \leq \frac{1}{2} \frac{d}{d t} z^{2} \leq\left(b+c_{1}\right) z^{2}, \quad z(0)=\|\nu\|
$$

and then

$$
\|\nu\| e^{a_{1} t} \leq z \leq\|\nu\| e^{b_{1} t} \quad \text { for any } t \geq 0 \text { and } \nu \in \mathbb{R}^{n} .
$$


Lemma 5. If the perturbation $Z_{1}$ fulfils the estimates

$$
\begin{aligned}
& \left\|D^{l} Z_{1}(x)\right\| \leq c_{l}^{\prime}\|x\|^{1-l+m} \quad \text { for any } x \in B(0,1) \text { and all integers } m \geq 1, \\
& \left\|D^{l} Z_{1}(x)\right\| \leq c_{l}^{\prime \prime}\|x\|^{1-l} \quad \forall x \in \mathbb{R}^{n} \backslash B(0,1)
\end{aligned}
$$

with $l=0,1$, then the first derivative of the $Y_{1}$-flow is complete and is estimated by, for any $t \geq 0$

$$
e^{a_{1} t} \leq\left\|D \psi_{t}^{1}(x)\right\| \leq e^{b_{1} t}, \quad e^{-b_{1} t} \leq\left\|D \psi_{-t}^{1}(x)\right\| \leq e^{-a_{1} t}
$$

with $c_{l}=\max \left\{c_{l}^{\prime}, c_{l}^{\prime \prime}\right\}, a_{l}=a-c_{l}$ and $b_{l}=b+c_{l}, l=0,1$.

Proof. For any $x \in B(0,1)$ we have $\left\|D^{l} Z_{1}(x)\right\| \leq c_{l}^{\prime}\|x\|^{1-l+m} \leq c_{l}^{\prime}\|x\|^{1-l}$ and letting $c_{l}=$ $\max \left\{c_{l}^{\prime}, c_{l}^{\prime \prime}\right\}$, we get for any $x \in \mathbb{R}^{n}\left\|D^{l} Z_{1}(x)\right\| \leq c_{l}\|x\|^{1-l}$. By the same arguments as in previous lemmas we get the estimates (18).

Lemma 6. Suppose that all the coefficients $\alpha_{i}$ are negative, $a \leq \alpha_{i} \leq b<0$.

If the perturbation $Z_{1}$ fulfills the estimates

$$
\left\|Z_{1}(x)\right\| \leq c_{0}\|x\|^{1+m}, \quad\left\|D Z_{1}(x)\right\| \leq c_{1}\|x\|^{m} \quad \text { for all } x \in \mathbb{R}^{n} \text { and any integers } m \geq 1 .
$$

Then the estimates of the first derivation of the $Y_{1}$-flow are as follows, for any $t \geq 0$

$$
e^{a t}\left(1-\frac{c_{0}}{a}\|x\|^{m}\left(1-e^{a m t}\right)\right)^{-\frac{c_{1}}{m c_{0}}} \leq\left\|D \psi_{t}^{1}(x)\right\| \leq e^{b t}\left(1-\frac{c_{0}}{b}\|x\|^{m}\left(1-e^{b m t}\right)\right)^{-\frac{c_{1}}{m c_{0}}} .
$$

Proof. Letting $y=\left\|\psi_{t}^{1}(x)\right\|$ and $z=\left\|\eta_{1}^{1}(t, x, \nu)\right\|$ in equation (16), we get

$$
\left(a-c_{1} y^{m}\right) z^{2} \leq \frac{1}{2} \frac{d}{d t} z^{2} \leq\left(b+c_{1} y^{m}\right) z^{2}, \quad z(0)=\|\nu\|
$$

and taking into account the estimates given by the relation (11), we obtain

$$
\|x\|^{m} e^{m a t}\left(1-\frac{c_{0}}{a}\|x\|^{m}\left(1-e^{a m t}\right)\right)^{-1} \leq y^{m} \leq\|x\|^{m} e^{m b t}\left(1-\frac{c_{0}}{b}\|x\|^{m}\left(1-e^{b m t}\right)\right)^{-1}
$$

consequently

$$
\begin{aligned}
& \|\nu\| \exp \left(a t-c_{1} \int_{0}^{t} \frac{\|x\|^{m} e^{m a s} d s}{1-\frac{c_{0}}{a}\|x\|^{m}\left(1-e^{a m s}\right)}\right) \\
& \quad \leq z \leq\|\nu\| \exp \left(b t+c_{1} \int_{0}^{t} \frac{\|x\|^{m} e^{m b s} d s}{1-\frac{c_{0}}{b}\|x\|^{m}\left(1-e^{b m s}\right)}\right)
\end{aligned}
$$

which has the solution

$$
\begin{aligned}
& \|\nu\| e^{a t}\left(1-\frac{c_{0}}{a}\|x\|^{m}\left(1-e^{a m t}\right)\right)^{-\frac{c_{1}}{m c_{0}}} \\
& \quad \leq z \leq\|\nu\| e^{b t}\left(1-\frac{c_{0}}{b}\|x\|^{m}\left(1-e^{b m t}\right)\right)^{-\frac{c_{1}}{m c_{0}}} \quad \text { for } \quad \nu \in \mathbb{R}^{n} .
\end{aligned}
$$

Example 2. We consider the same vector field as in Example 1. Denote by $\xi_{3}^{1}(t, x, \nu)=$ $D \phi_{t}^{3}(x) \nu, \forall \nu \in \mathbb{R}^{n}$, the first derivation of the $X_{3}$-flow. In coordinates, we have for any $i, j=$ $1, \ldots, n$,

$$
\left(\phi_{t}^{3}(x)\right)_{i}=x_{i} e^{\alpha_{i} t}\left(1+\frac{\beta_{i}}{\alpha_{i}} x_{i}^{m_{i}}\left(1-e^{\alpha_{i} m_{i} t}\right)\right)^{\frac{-1}{m_{i}}}
$$


so we deduce that

$$
\frac{\partial}{\partial x_{j}}\left(\phi_{t}^{3}(x)\right)_{i}=e^{\alpha_{i} t}\left(1+\frac{\beta_{i}}{\alpha_{i}} x_{i}^{m_{i}}\left(1-e^{\alpha_{i} m_{i} t}\right)\right)^{-1-\frac{1}{m_{i}}} \delta_{j}^{i}
$$

and by the estimates (13) we get

$$
e^{a t} \leq\left\|D \phi_{t}^{3}(x)\right\| \leq e^{b t} .
$$

The second derivative is

$$
\frac{\partial^{2}}{\partial x_{i}^{2}}\left(\phi_{t}^{3}(x)\right)_{i}=-\left(1+m_{i}\right) \frac{\beta_{i}}{\alpha_{i}} x_{i}^{-1+m_{i}} e^{\alpha_{i} t}\left(1-e^{\alpha_{i} m_{i} t}\right)\left(1+\frac{\beta_{i}}{\alpha_{i}} x_{i}^{m_{i}}\left(1-e^{\alpha_{i} m_{i} t}\right)\right)^{-2-\frac{1}{m_{i}}} .
$$

Consequently, for $l=1,2$ and any $x \in B(0, \rho)$ with $\rho>0$ arbitrary fixed, there are constants $M_{l}>0$ such that

$$
\left\|D^{l} \phi_{t}^{3}(x)\right\| \leq M_{l} e^{b t} .
$$

\subsection{Perturbation of a nonlinear vector field}

Consider the nonlinear vector field

$$
X_{2}=\sum_{i=1}^{n} \beta_{i} x_{i}^{1+m_{i}} \frac{\partial}{\partial x_{i}} \quad \text { with all } m_{i}>0 \text { and all } \beta_{i} \leq 0 .
$$

The explicit form of the $X_{2}$-flow is then given by

$$
\phi_{t}^{2}(x)=x\left(1-m \beta t x^{m}\right)^{\frac{-1}{m}}
$$

for any $t \geq 0$ in the sense

$$
\left(\phi_{t}^{2}(x)\right)_{i}=x_{i}\left(1-m_{i} \beta_{i} t x_{i}^{m_{i}}\right)^{\frac{-1}{m_{i}}}, \quad 1 \leq i \leq n .
$$

Lemma 7. If the following assumptions are true

i) all the coefficients $\beta_{i}$ are non positive, $-a^{\prime} \leq \beta_{i} \leq-b^{\prime} \leq 0$

ii) all the exponents $m_{i}$ are even positive integers; $0<m_{0} \leq m_{i} \leq m_{0}^{\prime}$.

Then the vector field $X_{2}$ is semi-complete and the $X_{2}$-flow satisfies the estimates

$$
\|x\|\left(1+b^{\prime} m_{0} t\|x\|^{m_{0}}\right)^{\frac{-1}{m_{0}}} \leq\left\|\phi_{t}^{2}(x)\right\| \leq\|x\|\left(1+a^{\prime} m_{0}^{\prime} t\|x\|^{m_{0}^{\prime}}\right)^{\frac{-1}{m_{0}^{\prime}}} \quad \text { for any } t \geq 0 .
$$

Proof. Clearly the flow $\phi_{t}^{2}=\exp \left(t X_{2}\right)$ given by (19) is semi-complete i.e. defined for all $t \geq 0$.

Consider the equation

$$
\frac{1}{2} \frac{d}{d t}\left\|\phi_{t}^{2}(x)\right\|^{2}=\left\langle\phi_{t}^{2}(x), \beta\left(\phi_{t}^{2}(x)\right)^{1+m}\right\rangle
$$

and put $y=\phi_{t}^{2}(x)$, then

$$
b^{\prime} y^{2+m_{0}} \leq \frac{1}{2} \frac{d}{d t} y^{2} \leq a^{\prime} y^{2+m_{0}^{\prime}}, \quad y(0)=\|x\|
$$

and we get the estimates given in (20). 


\subsection{Estimation of the $k^{\text {th }}$ order derivation of the $X_{2}$-flow}

Let $\xi_{2}^{1}(t, x, \nu)=D \phi_{t}^{2}(x) \nu, \forall \nu \in \mathbb{R}^{n}$ be the first derivation of the $X_{2}$-flow.

By formula (19), we get in coordinates

$$
\frac{\partial}{\partial x_{j}}\left(\phi_{t}^{2}(x)\right)_{i}=\left(1-m_{i} \beta_{i} t x_{i}^{m_{i}}\right)^{-1-\frac{1}{m_{i}}} \delta_{i}^{j} \quad \text { with } \quad \delta_{i}^{j}= \begin{cases}1 & \text { if } i=j, \\ 0 & \text { if } i \neq j,\end{cases}
$$

where $i, j=1, \ldots, n$.

Consequently

$$
\left(1+b^{\prime} m t\|x\|^{m_{0}}\right)^{-1-\frac{1}{m_{0}}} \leq\left\|D \phi_{t}^{2}(x)\right\| \leq\left(1+a^{\prime} m_{0}^{\prime} t\|x\|^{m_{0}^{\prime}}\right)^{-1-\frac{1}{m_{0}^{\prime}}} .
$$

To get the estimates of the second derivative, we put

$$
w_{i}=1-m_{i} \beta_{i} t x_{i}^{m_{i}}
$$

so

$$
\frac{d}{d x_{i}} w_{i}=m_{i}\left(w_{i}-1\right) x_{i}^{-1} \quad \text { and } \quad \frac{\partial}{\partial x_{i}}\left(\phi_{t}^{2}(x)\right)_{i}=w_{i}^{-1-\frac{1}{m_{i}}} .
$$

Consequently

$$
\frac{\partial^{2}}{\partial x_{i}^{2}}\left(\phi_{t}^{2}(x)\right)_{i}=\left(1+m_{i}\right) x_{i}^{-1} w_{i}^{-\frac{1}{m_{i}}}\left(w_{i}^{-2}-w_{i}^{-1}\right)=x_{i}^{-1} w_{i}^{-\frac{1}{m_{i}}}\left(\frac{a_{1}^{2}}{w_{i}}+\frac{a_{2}^{2}}{w_{i}^{2}}\right),
$$

where $a_{1}^{2}$ and $a_{2}^{2}$ are real constants. Let $\rho>0$ be any arbitrary and fixed real number, then for any $x \in B(0, \rho)$ and any $t \geq t_{0}>0$ and $l=1,2$ there is $M_{l}>0$ such that

$$
\left\|D^{l} \phi_{t}^{2}(x)\right\| \leq M_{l} t^{-1-\frac{1}{m_{0}^{\prime}}} .
$$

Suppose that for $l=1, \ldots, k-1$, with fixed $k$, there exist constants $a_{j}^{l}$ and $M_{l}>0$ such that

$$
\frac{\partial^{l}}{\partial x_{i}^{l}}\left(\phi_{t}^{2}(x)\right)_{i}=x_{i}^{1-l} w_{i}{ }^{-\frac{1}{m_{i}}} \sum_{j=1}^{l} \frac{a_{j}^{l}}{w_{i}^{j}},
$$

where $a_{j}^{l}$ are real constants and

$$
\left\|D^{l} \phi_{t}^{2}(x)\right\| \leq M_{l} t^{-1-\frac{1}{m_{0}^{\prime}}} \quad \forall t>0 .
$$

For the estimates of the $k^{\text {th }}$ derivative, we compute

$$
\begin{aligned}
& \frac{\partial^{k}}{\partial x_{i}^{k}}\left(\phi_{t}^{2}(x)\right)_{i}=x_{i}^{1-k} w_{i}{ }^{-\frac{1}{m_{i}}} \sum_{j=1}^{k} \frac{a_{j}^{k}}{w_{i}^{j}}, \\
& \frac{\partial^{k}}{\partial x_{i}^{k}}\left(\phi_{t}^{2}(x)\right)_{i}=\frac{d}{d x_{i}} x_{i}^{2-k} w_{i}{ }^{-\frac{1}{m_{i}}} \sum_{j=1}^{k-1} \frac{a_{j}^{k-1}}{w_{i}^{j}} \\
& \quad=x_{i}^{1-k} w_{i}-\frac{1}{m_{i}} \sum_{j=1}^{k-1}\left(\frac{a_{j}^{k-1}}{w_{i}^{j}}\left(1-k-j m_{i}\right)+\frac{a_{j}^{k-1}}{w_{i}^{j+1}}\left(1+j m_{i}\right)\right)=x_{i}^{1-k} w_{i}^{-\frac{1}{m_{i}}} \sum_{j=1}^{k} \frac{a_{j}^{k}}{w_{i}^{j}},
\end{aligned}
$$

where $a_{j}^{k}$ are real constants.

So we resume 


\section{Proposition 2. Suppose that}

i) all the coefficients satisfy $\beta_{i} \leq 0,-a^{\prime} \leq \beta_{i} \leq-b^{\prime}$,

ii) the exponents $m_{i}$ are even natural numbers such that $0<m_{0} \leq m_{i} \leq m_{0}^{\prime}$.

Let $\rho>0$ be any arbitrary fixed real number. For any $x \in B(0, \rho)$, for any $t \geq t_{0}>0$ and $\forall k \geq 1$ there exist a constant $M_{k}>0$ such that

$$
\left\|D^{k} \phi_{t}^{2}(x)\right\| \leq M_{k} t^{-1-\frac{1}{m_{0}^{\prime}}}
$$

\subsection{Estimates of the $Y_{2}$-flow}

Let

$$
Y_{2}=\sum_{i=1}^{n}\left(\beta_{i} x_{i}^{1+m_{i}}+Z_{2 i}(x)\right) \frac{\partial}{\partial x_{i}}
$$

the perturbation of the nonlinear vector field $X_{2}$ and denote by $\psi_{t}^{2}=\exp \left(t Y_{2}\right)$ the solution of the dynamic system

$$
\frac{d}{d t} \psi_{t}^{2}(x)=Y_{2} \circ \psi_{t}^{2}(x), \quad \psi_{0}^{2}(x)=x .
$$

In coordinates we have, $i=1, \ldots, n$,

$$
\frac{\partial}{\partial t} \psi_{2, i}(t, x)=\beta_{i} \psi_{2, i}^{1+m_{i}}(t, x)+Z_{2 i}\left(\psi_{t}^{2}(x)\right), \quad \psi_{2, i}(0, x)=x_{i}
$$

Putting

$$
y_{i}(t)=\psi_{2, i}^{-m_{i}}(t, x)
$$

and

$$
\psi_{t}^{2}(x)=y^{\frac{-1}{m}}(t)=\left(y_{1}^{\frac{-1}{m_{1}}}(t), \ldots, y_{n}^{\frac{-1}{m_{n}}}(t)\right)
$$

we get

$$
y_{i}^{\prime}(t)=-m_{i} \psi_{2, i}^{-1-m_{i}}(t, x) \frac{\partial}{\partial t} \psi_{2, i}(t, x) .
$$

The Cauchy problem reads as

$$
y_{i}^{\prime}(t)=-m_{i} \beta_{i}-m_{i}\left(y_{i}(t)\right)^{1+\frac{1}{m_{i}}} Z_{2 i}\left(y^{\frac{-1}{m}}(t)\right), \quad y_{i}(0)=x_{i}^{-m_{i}}
$$

and has the following solution

$$
y_{i}(t)=x_{i}^{-m_{i}}-m_{i} \beta_{i} t-m_{i} \int_{0}^{t} y_{i}(s)^{1+\frac{1}{m_{i}}} Z_{2 i}\left(y^{\frac{-1}{m}}(s) d s,\right.
$$

i.e.

$$
\psi_{2, i}(t, x)=x_{i}\left(1-m_{i} \beta_{i} t x_{i}^{m_{i}}-m_{i} x_{i}^{m_{i}} \int_{0}^{t} \psi_{i}(s, x)^{-1-m_{i}} Z_{2 i}\left(\psi_{s}^{2}(x)\right) d s\right)^{-\frac{1}{m_{i}}},
$$

so we have the explicit form of the $Y_{2}$-flow

$$
\psi_{t}^{2}(x)=x\left(1-m \beta t x^{m}-m x^{m} \int_{0}^{t} \psi_{s}^{2}(x)^{-1-m} Z_{2}\left(\psi_{s}^{2}(x)\right) d s\right)^{-\frac{1}{m}} .
$$

Now we will estimate the $Y_{2}$-flow. 
Lemma 8. Suppose that

i) all the coefficients satisfy $\beta_{i} \leq 0,-a^{\prime} \leq \beta_{i} \leq-b^{\prime}$;

ii) the exponents $m_{i}$ are even natural numbers with $0<m_{0} \leq m_{i} \leq m_{0}^{\prime}$;

iii)

$$
\begin{array}{ll}
\left\|Z_{2 i}(x)\right\| \leq c_{0}^{\prime}\left|x_{i}\right|^{2+m_{i}} & \text { if } x \in B(0,1), \\
\left\|Z_{2 i}(x)\right\| \leq c_{0}^{\prime \prime}\left|x_{i}\right|^{1+m_{i}} & \text { if } x \in \mathbb{R}^{n} \backslash B(0,1)
\end{array}
$$

with $c_{0}=\max \left\{c_{0}^{\prime}, c_{0}^{\prime \prime}\right\}, b_{0}=b^{\prime}-c_{0}>0, a_{0}=a^{\prime}+c_{0}$.

Then

1) the vector field $Y_{2}$ is semi-complete;

2) the $Y_{2}$-flow has the estimates

$$
\|x\|\left(1+a_{0} m_{0} t\|x\|^{m_{0}}\right)^{\frac{-1}{m_{0}}} \leq\left\|\psi_{t}^{2}(x)\right\| \leq\|x\|\left(1+b_{0} m_{0}^{\prime} t\|x\|^{m_{0}^{\prime}}\right)^{\frac{-1}{m_{0}^{\prime}}} ;
$$

3) let $\rho>0$ and $t_{0}>0$ be fixed, then for any $x \in B(0, \rho)$ and any $t \geq t_{0}>0$ there is a constant $M_{0}>0$ such that

$$
\left\|\psi_{t}^{2}(x)\right\| \leq M_{0}\|x\| t^{-\frac{1}{m_{0}^{\prime}}}
$$

Proof. Let $x \in B(0,1)$, by assumption we have $\left\|Z_{2 i}(x)\right\| \leq c_{0}^{\prime}\left|x_{i}\right|^{2+m_{i}} \leq c_{0}^{\prime}\left|x_{i}\right|^{1+m_{i}}$, put $c_{0}=\max \left\{c_{0}^{\prime}, c_{0}^{\prime \prime}\right\}$ then for any $x \in \mathbb{R}^{n}$ we deduce $\left\|Z_{2 i}(x)\right\| \leq c_{0}\left|x_{i}\right|^{1+m_{i}}$. Now taking account of the relation (23) we deduce that for any $t \in[0, T]$

$$
\left\|\psi_{t}^{2}(x)\right\| \leq\|x\|\left(1+m t\|x\|^{m}\left(b^{\prime}-c_{0}\right)\right)^{-\frac{1}{m}} \leq\|x\|
$$

hence the vector $Y_{2}$ is semi-complete, i.e. defined for all $t \geq 0$.

Consider the equation

$$
\frac{1}{2} \frac{d}{d t}\left\|\left(\psi_{t}^{2}(x)\right)_{i}\right\|^{2}=\left\langle\left(\psi_{t}^{2}(x)\right)_{i}, \beta_{i}\left(\psi_{t}^{2}(x)\right)_{i}^{1+m_{i}}+Z_{2 i}\left(\psi_{t}^{2}(x)\right)\right\rangle
$$

we get $y_{i}=\left\|\left(\psi_{t}^{2}(x)\right)_{i}\right\|$ and $y_{i}(0)=\left|x_{i}\right|$, so we deduce

$$
\frac{1}{2} \frac{d}{d t} y_{i}^{2} \leq\left(\beta_{i}+c_{0}\right) y_{i}^{2+m_{i}} \leq-\left(b^{\prime}-c_{0}\right) y_{i}^{2+m_{i}}
$$

and

$$
\frac{1}{2} \frac{d}{d t} y_{i}^{2} \geq\left(\beta_{i}-c_{0}\right) y_{i}^{2+m_{i}} \geq-\left(a^{\prime}+c_{0}\right) y_{i}^{2+m_{i}}
$$

We put $b_{0}=b^{\prime}-c_{0}$ and $a_{0}=a^{\prime}+c_{0}$, the solutions are estimated as

$$
\left(\left|x_{i}\right|^{-m_{i}}+a_{0} m_{i} t\right)^{-\frac{1}{m_{i}}} \leq\left\|\left(\psi_{t}^{2}(x)\right)_{i}\right\| \leq\left(\left|x_{i}\right|^{-m_{i}}+b_{0} m_{i} t\right)^{-\frac{1}{m_{i}}}
$$

Hence, we have the estimate (25).

Now, we estimate the first derivation of the $Y_{2}$-flow. Let $\eta_{2}^{1}(t, x, \nu)=D \psi_{t}^{2}(x) \nu, \forall \nu \in \mathbb{R}^{n}$ the solution of the dynamic system

$$
\frac{d}{d t} \eta_{2}^{1}(t, x, \nu)=\left(D_{y} X_{2}+D_{y} Z_{2}\right) \eta_{2}^{1}(t, x, \nu), \quad \eta_{2}^{1}(0, x, \nu)=\nu
$$

with $y=\psi_{t}^{2}(x)$. 
Lemma 9. Suppose that

i) the coefficients are such that $\beta_{i} \leq 0,-a^{\prime} \leq \beta_{i} \leq-b^{\prime}$;

ii) the coefficients $m_{i}$ are even natural numbers, $0<m_{0} \leq m_{i} \leq m_{0}^{\prime}$;

iii)

$$
\begin{array}{ll}
\left\|D^{l} Z_{2 i}(x)\right\| \leq c_{l}^{\prime}\left|x_{i}\right|^{2-l+m_{i}} & \text { if } x \in B(0,1), \\
\left\|D^{l} Z_{2 i}(x)\right\| \leq c_{l}^{\prime \prime}\left|x_{i}\right|^{1-l+m_{i}} & \text { if } x \in \mathbb{R}^{n} \backslash B(0,1)
\end{array}
$$

with $l=0,1$;

$i v)$

$$
a_{0}=a^{\prime}+c_{0}, \quad b_{0}=b^{\prime}-c_{0}>0
$$

and

$$
a_{1}=a^{\prime}\left(1+m_{0}\right)+c_{1}, \quad b_{1}=b^{\prime}\left(1+m_{0}\right)-c_{1}>0
$$

with $c_{l}=\max \left\{c_{l}^{\prime}, c_{l}^{\prime \prime}\right\}$.

Then the first derivation of the $Y_{2}$-flow has the following estimates, for any $t>0$

$$
\left(1+b_{0} m_{0} t\|x\|^{m_{0}}\right)^{-\frac{a_{1}}{b_{0} m_{0}}} \leq\left\|D \psi_{t}^{2}(x)\right\| \leq\left(1+a_{0} m_{0}^{\prime} t\|x\|^{m_{0}^{\prime}}\right)^{-\frac{b_{1}}{a_{0} m_{0}^{\prime}}} .
$$

Let $\rho>0$ be arbitrary and fixed for any $x \in B(0, \rho)$, and any $t \geq t_{0}>0$ there is a constant $M_{1}>0$ such that

$$
\left\|D \psi_{t}^{2}(x)\right\| \leq M_{1} t^{-\frac{b_{1}}{a_{0} m_{0}^{\prime}}} .
$$

Proof. Let $x \in B(0,1)$, for $l=0,1$ we have

$$
\left\|D^{l} Z_{2 i}(x)\right\| \leq c_{l}^{\prime}\left|x_{i}\right|^{2-l+m_{i}} \leq c_{l}^{\prime}\left|x_{i}\right|^{1-l+m_{i}} .
$$

Let $c_{l}=\max \left\{c_{l}^{\prime}, c_{l}^{\prime \prime}\right\}$ then for $x \in \mathbb{R}^{n}$ one has

$$
\left\|D^{l} Z_{2 i}(x)\right\| \leq c_{l}\left|x_{i}\right|^{1-l+m_{i}} .
$$

Consider the equation

$$
\frac{1}{2} \frac{d}{d t}\left\|\eta_{2}^{1}(t, x, \nu)\right\|^{2}=\left\langle\eta_{2}^{1}(t, x, \nu),\left(D_{y} X_{2}+D_{y} Z_{2}\right) \eta_{2}^{1}(t, x, \nu)\right\rangle
$$

and put $z(t)=\left\|\eta_{2}^{1}(t, x, \nu)\right\|$ with $z(0)=\|\nu\|$, then

$$
\frac{1}{2} \frac{d}{d t} z^{2} \leq \sup _{i=1, \ldots, n}\left(\left(\left(1+m_{i}\right) \beta_{i}+c_{1}\right)\left\|\left(\psi_{t}^{2}(x)\right)_{i}\right\|^{m_{i}}\right) z^{2} \leq z^{2} \sup _{i=1, \ldots, n}\left(-b_{1}\left\|\left(\psi_{t}^{2}(x)\right)_{i}\right\|^{m_{i}}\right)
$$

and

$$
\frac{1}{2} \frac{d}{d t} z^{2} \geq \inf _{i=1, \ldots, n}\left(\left(\left(1+m_{i}\right) \beta_{i}-c_{1}\right)\left\|\left(\psi_{t}^{2}(x)\right)_{i}\right\|^{m_{i}}\right) z^{2} \geq z^{2} \inf _{i=1, \ldots, n}\left(-a_{1}\left\|\left(\psi_{t}^{2}(x)\right)_{i}\right\|^{m_{i}}\right) .
$$

The solutions fulfill the following estimates

$$
\begin{aligned}
& \|\nu\| \exp \inf _{i=1, \ldots, n}\left(-a_{1} \int_{0}^{t}\left\|\left(\psi_{s}^{2}(x)\right)_{i}\right\|^{m_{i}} d s\right) \\
& \leq z(t) \leq\|\nu\| \exp \sup _{i=1, \ldots, n}\left(-b_{1} \int_{0}^{t}\left\|\left(\psi_{s}^{2}(x)\right)_{i}\right\|^{m_{i}} d s\right)
\end{aligned}
$$


with, by (26)

$$
\frac{\left|x_{i}\right|^{m_{i}}}{1+a_{0} m_{i} t\left|x_{i}\right|^{m_{i}}} \leq\left\|\left(\psi_{t}^{2}(x)\right)_{i}\right\|^{m_{i}} \leq \frac{\left|x_{i}\right|^{m_{i}}}{1+b_{0} m_{i} t\left|x_{i}\right|^{m_{i}}} .
$$

So we deduce

$$
\begin{aligned}
& \|\nu\| \exp \inf _{i=1, \ldots, n}\left(-a_{1} \int_{0}^{t} \frac{\left|x_{i}\right|^{m_{i}}}{1+b_{0} m_{i} s\left|x_{i}\right|^{m_{i}}} d s\right) \\
& \quad \leq z(t) \leq\|\nu\| \exp \sup _{i=1, \ldots, n}\left(-b_{1} \int_{0}^{t} \frac{\left|x_{i}\right|^{m_{i}}}{1+a_{0} m_{i} s\left|x_{i}\right|^{m_{i}}} d s\right) .
\end{aligned}
$$

Consequently the solutions satisfy

$$
\|\nu\| \inf _{i=1, \ldots, n}\left(1+b_{0} m_{i} t\left|x_{i}\right|^{m_{i}}\right)^{-\frac{a_{1}}{b_{0} m_{i}}} \leq z(t) \leq\|\nu\| \sup _{i=1, \ldots, n}\left(1+a_{0} m_{i} t\left|x_{i}\right|^{m_{i}}\right)^{-\frac{b_{1}}{a_{0} m_{i}}} .
$$

Then there are constants $m_{0}>0$ and $m_{0}^{\prime}>0$ such that

$$
\begin{aligned}
& \|\nu\|\left(1+b_{0} m_{0} t\|x\|^{m_{0}}\right)^{-\frac{a_{1}}{b_{0} m_{0}}} \leq\left\|D \psi_{t}^{2}(x) \nu\right\| \\
& \quad \leq\|\nu\|\left(1+a_{0} m_{0}^{\prime} t\|x\|^{m_{0}^{\prime}}\right)^{-\frac{b_{1}}{a_{0} m_{0}^{\prime}}} \quad \forall \nu \in \mathbb{R}^{n} \text { and for any } t>0 .
\end{aligned}
$$

Hence, we have the estimate (28).

\subsection{Perturbation of binomial vector fields}

Let

$$
Y_{3}=\sum_{i=1}^{n}\left(\alpha_{i} x_{i}+\beta_{i} x_{i}^{1+m_{i}}+Z_{3 i}(x)\right) \frac{\partial}{\partial x_{i}}
$$

with $a \leq \alpha_{i} \leq b<0, a^{\prime} \leq \beta_{i} \leq b^{\prime} \leq 0$ and $0<m_{0} \leq m_{i} \leq m_{0}^{\prime}$, be the perturbation of the binomial vector field $X_{3}$ and let $\psi_{t}^{3}=\exp \left(t Y_{3}\right)$ be the $Y_{3}$-flow which is the solution of the dynamic system

$$
\frac{d}{d t} \psi_{t}(x)=Y_{3} \circ \psi_{t}(x), \quad \psi_{0}(x)=x
$$

and in coordinates, we get

$$
\frac{\partial}{\partial t} \psi_{3, i}(t, x)=\alpha_{i} \psi_{3, i}(t, x)+\beta_{i} \psi_{3, i}^{1+m_{i}}(t, x)+Z_{3, i}\left(\psi_{t}^{3}(x)\right), \quad \psi_{i}(0, x)=x_{i}
$$

which is a Bernoulli type equation and by the same method as in the proof of previous lemmas and with putting

$$
y_{i}(t)=\psi_{3, i}^{-m_{i}}(t, x)
$$

and

$$
\psi_{t}^{3}(x)=y^{\frac{-1}{m}}(t)=\left(y_{1}^{\frac{-1}{m_{1}}}(t), \ldots, y_{n}^{\frac{-1}{m_{n}}}(t)\right)
$$


we get the solution

$$
\begin{aligned}
\psi_{3, i}(t, x)= & x_{i} e^{\alpha_{i} t}\left(1+\frac{\beta_{i}}{\alpha_{i}} x_{i}^{m_{i}}\left(1-e^{\alpha_{i} m_{i} t}\right)\right. \\
& \left.-m_{i} x_{i}^{m_{i}} \int_{0}^{t}\left[\psi_{3, i}(s, x)\right]^{-1-m_{i}} Z_{3, i}\left(\psi_{s}^{3}(x)\right) e^{\alpha_{i} m_{i} s} d s\right)^{\frac{-1}{m_{i}}}
\end{aligned}
$$

and the implicit form of the $Y_{3}$-flow reads as

$$
\psi_{t}^{3}(x)=x e^{\alpha t}\left(1+\frac{\beta}{\alpha} x^{m}\left(1-e^{\alpha m t}\right)-m x^{m} \int_{0}^{t}\left[\psi_{s}^{3}(x)\right]^{-1-m} Z_{3}\left(\psi_{s}^{3}(x)\right) e^{\alpha m s} d s\right)^{-\frac{1}{m}} .
$$

\subsection{Estimation of the $Y_{3}$-flow}

By the same arguments as in the previous, we get the following estimates of the $Y_{3}$-flow.

Lemma 10. If the following assumptions are true

i) all the coefficients $\alpha_{i}$ are negative, $-a \leq \alpha_{i} \leq-b<0$;

ii) all the coefficients $\beta_{i}$ are non positive, $-a^{\prime} \leq \beta_{i} \leq-b^{\prime}$;

iii) the exponents $m_{i}$ are even natural numbers with $0<m_{0} \leq m_{i} \leq m_{0}^{\prime}$;

$i v)$

$$
\begin{aligned}
& \left\|Z_{3 i}(x)\right\| \leq c_{0}^{\prime}\left|x_{i}\right|^{2+m_{i}} \quad \text { if } x \in B(0,1), \\
& \left\|Z_{3 i}(x)\right\| \leq c_{0}^{\prime \prime}\left|x_{i}\right|^{1+m_{i}} \quad \text { if } x \in \mathbb{R}^{n} \backslash B(0,1)
\end{aligned}
$$

with $c_{0}=\max \left\{c_{0}^{\prime}, c_{0}^{\prime \prime}\right\}, b_{0}=b^{\prime}-c_{0}>0, a_{0}=a^{\prime}+c_{0}$.

Then

1) there exist constants $m>0$ and $m^{\prime}>0$ such that the $Y_{3}$-flow has the estimates, $\forall t \geq 0$

$$
\begin{aligned}
& \|x\| e^{-a t}\left(1+\frac{a_{0}}{a}\|x\|^{m}\left(1-e^{-a m t}\right)\right)^{-\frac{1}{m}} \\
& \quad \leq\left\|\psi_{t}^{3}(x)\right\| \leq\|x\| e^{-b t}\left(1+\frac{b_{0}}{b}\|x\|^{m^{\prime}}\left(1-e^{-b m^{\prime} t}\right)\right)^{-\frac{1}{m^{\prime}}}
\end{aligned}
$$

2) for any $t>0$ there are positive constants $c_{1}$ and $c_{2}$ such that

$$
c_{1}\|x\| e^{-a t} \leq\left\|\psi_{t}^{3}(x)\right\| \leq c_{2}\|x\| e^{-b t} ;
$$

3) the vector field $Y_{3}$ is semi-complete.

By similar calculations as in previous lemmas, we get the following estimates to the first derivative of the $Y_{3}$-flow.

Lemma 11. Suppose that

i) all the coefficients $\alpha_{i}$ are negative, $-a \leq \alpha_{i} \leq-b<0$;

ii) all the coefficients $\beta_{i}$ are non positive, $-a^{\prime} \leq \beta_{i} \leq-b^{\prime}$;

iii) the exponents $m_{i}$ are even natural numbers such that $0<m_{0} \leq m_{i} \leq m_{0}^{\prime}$;

$i v)$

$$
\begin{array}{ll}
\left\|D^{l} Z_{3 i}(x)\right\| \leq c_{l}^{\prime}\left|x_{i}\right|^{2-l+m_{i}} & \text { if } x \in B(0,1), \\
\left\|D^{l} Z_{3 i}(x)\right\| \leq c_{l}^{\prime \prime}\left|x_{i}\right|^{1-l+m_{i}} & \text { if } x \in \mathbb{R}^{n} \backslash B(0,1)
\end{array}
$$

with $l=0,1$; 
$v)$

$$
a_{0}=a^{\prime}+c_{0}, \quad b_{0}=b^{\prime}-c_{0}>0
$$

and

$$
a_{1}=a^{\prime}\left(1+m_{0}\right)+c_{1}, \quad b_{1}=b^{\prime}\left(1+m_{0}\right)-c_{1}>0
$$

with $c_{l}=\max \left\{c_{l}^{\prime}, c_{l}^{\prime \prime}\right\}$.

Then there exist constants $m>0$ and $m^{\prime}>0$ such that for any $t \geq 0$

$$
\begin{aligned}
& e^{-a t}\left(1+\frac{b_{0}}{b}\|x\|^{m}\left(1-e^{-b m t}\right)\right)^{-\frac{a_{1}}{b_{0} m}} \\
& \quad \leq\left\|D \psi_{t}^{3}(x)\right\| \leq e^{-b t}\left(1+\frac{a_{0}}{a}\|x\|^{m^{\prime}}\left(1-e^{-a m^{\prime} t}\right)\right)^{-\frac{b_{1}}{a_{0} m^{\prime}}}
\end{aligned}
$$

and for any $t \geq 0$, there is a constant $M_{1}>0$ such that

$$
\left\|D \psi_{t}^{3}(x)\right\| \leq M_{1} e^{-b t} .
$$

\section{Global stability of prolongations of flows}

With notations of the previous sections, we will give global stability of some flows.

\subsection{Global stability of the $Y_{1}$-flow}

Lemma 12. Let the vector fields

$$
Y_{1}=\sum_{i=1}^{n}\left(\alpha_{i} x_{i}+Z_{1 i}(x)\right) \frac{\partial}{\partial x_{i}}
$$

with the following assumptions

i) all the coefficients are negative, $-a \leq \alpha_{i} \leq-b<0$;

ii)

$$
\begin{aligned}
& \left\|Z_{1}(x)\right\| \leq c_{0}^{\prime}\|x\|^{1+m} \quad \forall x \in B(0,1) \quad \text { and } \forall m \geq 1, \\
& \left\|Z_{1}(x)\right\| \leq c_{0}^{\prime \prime}\|x\| \quad \forall x \in \mathbb{R}^{n} \backslash B(0,1) ;
\end{aligned}
$$

iii) $b_{0}=b-c_{0}>0$, where $c_{0}=\max \left\{c_{0}^{\prime}, c_{0}^{\prime \prime}\right\}$.

Then the origin 0 is a globally asymptotically stable equilibrium to the $Y_{1}$-flow $\psi_{t}^{1}$ on $\mathbb{R}^{n}$.

Proof. Let $\psi_{t}^{1}=\exp \left(t Y_{1}\right)$ be the $Y_{1}$-flow, then by the assumptions and the estimates given by Lemma 2 we get that

$$
\left\|\psi_{t}^{1}(x)\right\| \leq\|x\| e^{-b_{0} t} \quad \forall t \geq 0 \text { and } \forall x \in \mathbb{R}^{n}
$$

and by Proposition 1, the origin 0 is G.A.S. for $\psi_{t}^{1}$ on $\mathbb{R}^{n}$.

Example 3. We consider the vector field

$$
X_{3}=\sum_{i=1}^{n}\left(\alpha_{i} x_{i}+\beta_{i} x_{i}^{1+m_{i}}\right) \frac{\partial}{\partial x_{i}}
$$


of Example 1 with $a \leq \alpha_{i} \leq b<0, a^{\prime} \leq \beta_{i} \leq b^{\prime} \leq 0$. The $X_{3}$-flow $\phi_{t}^{3}=\exp \left(t X_{3}\right)$ is then given by

$$
\phi_{t}^{3}(x)=x e^{\alpha t}\left(1+\frac{\beta}{\alpha} x^{m}\left(1-e^{\alpha m t}\right)\right)^{\frac{-1}{m}} .
$$

Let $\rho>0$ be arbitrary and fixed real number. By the estimates (13), we have for any $x \in B(0, \rho)$ and any $t \geq t_{0} \geq 0$

$$
\left\|\phi_{t}^{3}(x)\right\| \leq\|x\| e^{-b t} \text {. }
$$

By Proposition1 the origin 0 is a G.A.S. for the flow $\phi_{t}^{3}$ on $\mathbb{R}^{n}$.

\subsection{Global stability of the first prolongation of the $Y_{1}$-flow}

Lemma 13. With the same assumptions as in Lemma 12 and the following conditions

$$
\begin{aligned}
& \left\|D Z_{1}(x)\right\| \leq c_{1}^{\prime}\|x\|^{m} \quad \forall x \in B(0,1) \quad \text { and } \forall m \geq 1, \\
& \left\|D Z_{1}(x)\right\| \leq c_{1}^{\prime \prime} \quad \forall x \in \mathbb{R}^{n} \backslash B(0,1)
\end{aligned}
$$

with $b_{1}=b-c_{1}>0$ and $c_{1}=\max \left\{c_{1}^{\prime}, c_{1}^{\prime \prime}\right\}$.

Then the origin 0 is a globally asymptotically stable for the first prolongation of the $Y_{1}$-flow $\psi_{t}^{1}$ on $\mathbb{R}^{n}$.

Proof. By the estimates (18) and the hypothesis we deduce that

$$
\left\|D \psi_{t}^{1}(x) \nu\right\| \leq\|\nu\| e^{-b_{1} t} \quad \forall t>0, \quad \forall \nu \in \mathbb{R}^{n}
$$

and by Proposition 1, we obtain that the origin 0 is a $G$.A.S. equilibrium on $\mathbb{R}^{n}$ for $\eta_{1}^{1}(t, x, v)=$ $D \psi_{t}^{1}(x) \nu$.

\subsection{Global stability of the $k^{\text {th }}$ prolongation of the $Y_{1}$-flow}

Suppose that

i) all the coefficients are negative, $-a \leq \alpha_{i} \leq-b<0$;

ii) for any $l=1, \ldots, k-1$

$$
\begin{aligned}
& \left\|D^{l} Z_{1}(x)\right\| \leq c_{l}^{\prime}\|x\|^{1-l+m} \quad \text { for any } x \in B(0,1) \text { and for any integer } m \geq l-1, \\
& \left\|D^{l} Z_{1}(x)\right\| \leq c_{l}^{\prime \prime} \quad \forall x \in \mathbb{R}^{n} \backslash B(0,1), \\
& a_{0}=a+c_{0}, \quad b_{0}=b-c_{0}>0, \\
& a_{1}=a+c_{1}, \quad b_{1}=b-c_{1}>0
\end{aligned}
$$

with $c_{l}=\max \left\{c_{l}^{\prime}, c_{l}^{\prime \prime}\right\}, b_{l}=c_{l} \forall l \geq 2$.

Put $\eta_{1}^{l}(t, x, \nu, \ldots, \nu)=D^{k} \psi_{t}^{1}(x) \nu^{k}$, where $\nu \in \mathbb{R}^{n}$. Since by Lemmas 12 and 13 the origin 0 is an G.A.S. equilibrium for $\eta_{1}^{l}$, with $l=0,1$, on $\mathbb{R}^{n}$, we suppose that this property remains true for $l=0,1, \ldots, k-1$ with $k \geq 2$ i.e. for any $\rho>0$ and any $x \in B(0, \rho)$ there exist constants $M_{l}>0$ such that for any $t \geq t_{0}>0$

$$
\left\|D^{l} \psi_{t}^{1}(x)\right\| \leq M_{l} e^{-b_{1} t} .
$$

We will show that the origin 0 is a G.A.S. equilibrium for $\eta_{1}^{k}$ on $\mathbb{R}^{n}$. $\eta_{1}^{k}(t, x, \nu, \ldots, \nu)=$ $D^{k} \psi_{t}^{1}(x) \nu^{k}$ is solution of the dynamic system

$$
\frac{d}{d t} \eta_{1}^{k}=D_{y} Y_{1} \cdot \eta_{1}^{k}+G_{1}^{k}(t, x, \nu), \quad \eta_{1}^{k}(0, x, \nu, \ldots, \nu)=\nu
$$


with $y=\psi_{t}^{1}(x)$ and

$$
\begin{aligned}
G_{1}^{k}(t, x, \nu) & =\sum_{l=2}^{k} D_{y}^{l} Y_{1}(y) \sum_{\substack{i_{1}+\cdots+i_{l}=k \\
i_{j}>0}}\left(\prod_{j=1}^{l} D^{i_{j}} \psi_{t}^{1}(x) \nu^{i_{j}}\right) \\
& =\sum_{l=2}^{k-1} D_{y}^{l} Z_{1}(y) \sum_{\substack{i_{1}+\cdots+i_{l}=k \\
i_{j}>0}}\left(\prod_{j=1}^{l} D^{i_{j}} \psi_{t}^{1}(x) \nu^{i_{j}}\right)+D_{y}^{k} Z_{1}(y)\left(D \psi_{t}^{1}(x) \nu\right)^{k} .
\end{aligned}
$$

Consequently we get

$$
\eta_{1}^{k}(t, x, \nu, \ldots, \nu)=D \psi_{t}^{1}(x) \nu+\int_{0}^{t} D \Psi_{t-s}^{1}\left(\psi_{s}^{1}(x)\right) G_{1}^{k}(s, x, \nu) d s .
$$

The integral is well defined at $s=0$, since

$$
\lim _{s \rightarrow 0^{+}} D \psi_{t-s}^{1}\left(\psi_{s}^{1}(x)\right)=D \psi_{t}^{1}(x)
$$

and there exist constants $A_{l}>0$ such that

$$
\lim _{s \rightarrow 0^{+}} G_{1}^{k}(s, x, \nu)=\sum_{l=2}^{k} A_{l} D_{y}^{l} Z_{1}(y) \nu^{k} .
$$

We will show that it converges uniformly with respect to $x$ as $t+\infty$. Put

$$
I_{k}=\int_{0}^{t}\left\|D \psi_{t-s}^{1}\left(\psi_{s}^{1}(x)\right)\right\|\left\|G_{1}^{k}(s, x, \nu)\right\| d s .
$$

Since $\left\|D^{l} Z_{1}(x)\right\| \leq c_{l} \forall l \geq 1, \forall x \in \mathbb{R}^{n}$, there are constants $b_{l}>0$ such that $\forall y \in \mathbb{R}^{n}$, $\left\|D_{y}^{l} Y_{1}(y)\right\| \leq b_{l}$ and by the assumption of recurrence there exist constants $M_{l}>0$ such that

$$
\left\|D^{l} \psi_{t}^{1}(x)\right\| \leq M_{l} e^{-b_{1} t} \quad \forall t \geq 0 .
$$

We deduce that there is a constant $C_{k}>0$ such that

$$
I_{k} \leq \sum_{l=2}^{k} b_{l} M_{l} \int_{0}^{t} e^{-b_{1}(t-s+s l)} d s \leq C_{k} e^{-b_{1} t} .
$$

So for any $x \in \mathbb{R}^{n}$ one has

$$
\lim _{t \rightarrow+\infty} I_{k} \leq \sum_{l=2}^{k} M_{l} b_{l}\|\nu\|^{l} \int_{0}^{+\infty} e^{-b_{1} s} d s=\frac{1}{b_{1}} \sum_{l=2}^{k} M_{l} b_{l}\|\nu\|^{l}
$$

and the integral $I_{k}$ is uniformly convergent with respect to $x \in \mathbb{R}^{n}$ as $t \rightarrow+\infty$. Consequently

$$
\lim _{t \rightarrow+\infty}\left\|\eta_{1}^{k}\right\|=\lim _{t \rightarrow+\infty}\left\|D \psi_{t}^{1}(x) \nu\right\|+\int_{0}^{+\infty} \lim _{t \rightarrow+\infty}\left\|D \psi_{t-s}^{1}\left(\psi_{s}^{1}(x)\right)\right\|\left\|G_{1}^{k}(s, x, \nu)\right\| d s=0
$$

and there is a constant $M_{k}^{\prime}>0$ such that

$$
\left\|\eta_{1}^{k}\right\| \leq\left\|D \psi_{t}^{1}(x) \nu\right\|+\int_{0}^{+\infty}\left\|D \psi_{t-s}^{1}\left(\psi_{s}^{1}(x)\right)\right\|\left\|G_{1}^{k}(s, x, \nu)\right\| d s \leq M_{k}^{\prime}\|\nu\|^{k} e^{-b_{1} t} .
$$

This show by Proposition 1 that the origin 0 is a G.A.S. equilibrium to $\eta_{1}^{k}$ on $\mathbb{R}^{n}$. We formulate our proving as follows

Proposition 3. Let $k \geq 0$ be any integer. The origin 0 is a G.A.S. equilibrium of order $k$ for the $Y_{1}$-flow and there is a constant $M_{k}>0$ such that $\forall t>0$

$$
\left\|D^{k} \psi_{t}^{1}(x)\right\| \leq M_{k} e^{-b_{1} t}, \quad\left\|D^{k} \psi_{-t}^{1}(x)\right\| \leq M_{k} e^{a_{1} t} .
$$




\section{Global stability of a flow generated by nonlinear perturbed vector fields}

First we will start with monomial vector fields.

\subsection{Global stability of the $X_{2}$-flow}

Let

$$
X_{2}=\sum_{i=1}^{n} \beta_{i} x_{i}^{1+m_{i}} \frac{\partial}{\partial x_{i}}
$$

with

(i) all the coefficients $\beta_{i} \leq 0$ such that $-a^{\prime} \leq \beta_{i} \leq-b^{\prime}$;

(ii) all the exponents $m_{i}$ are even natural integers with $0<m_{0} \leq m_{i} \leq m_{0}^{\prime}$.

Let $\phi_{t}^{2}=\exp \left(t X_{2}\right)$ be the $X_{2}$-flow. By the estimations (19) we obtain

$$
\left\|\phi_{t}^{2}(x)\right\| \leq\|x\|\left(1+a^{\prime} m_{0}^{\prime} t\|x\|^{m_{0}^{\prime}}\right)^{\frac{-1}{m_{0}^{\prime}}} .
$$

Let $\rho>0$ be arbitrary fixed, for any $x \in B(0, \rho)$ and any $t \geq t_{0}>0$ there is a constant $M_{0}>0$ such that

$$
\left\|\phi_{t}^{2}(x)\right\| \leq M_{0}\|x\| t^{-\frac{1}{m_{0}^{\prime}}} .
$$

By Proposition 1, the origin is a globally asymptotically stable equilibrium to the flow $\phi_{t}^{2}$ on $\mathbb{R}^{n}$.

Let $l=1,2, \ldots$ any positive integer. By Proposition 2, we have: for any fixed $\rho>0$, and all $x \in B(0, \rho)$ and $t \geq t_{0}>0$, there exist constants $M_{l}>0$ and $M_{l}^{\prime}>0$ such that

$$
\left\|D^{l} \phi_{t}^{2}(x)\right\| \leq M_{l} t^{-1-\frac{1}{m_{0}^{\prime}}} \quad \text { and } \quad\left\|D^{l} \phi_{0}^{2}(x)\right\| \leq M_{l}^{\prime}
$$

So the origin 0 is a G.A.S. equilibrium for $D^{l} \phi_{t}^{2}(x)$ on $\mathbb{R}^{n}$.

Resuming our proving, we get

Proposition 4. Let $k \geq 0$ be any integer. Under the above conditions $(i)$ and $(i i)$, the origin 0 is a G.A.S. of order $k$ for the $X_{2}$-flow on $\mathbb{R}^{n}$.

\subsection{Global stability of high order of the $Y_{2}$-flow}

Let

$$
Y_{2}=\sum_{i=1}^{n}\left(\beta_{i} x_{i}^{1+m_{i}}+Z_{2, i}(x)\right) \frac{\partial}{\partial x_{i}}
$$

be a smooth vector field on $\mathbb{R}^{n}$ such that

i) all the coefficients $\beta_{i} \leq 0$ are non negative with $-a^{\prime} \leq \beta_{i} \leq-b^{\prime}$;

ii) $m_{i}$ are even natural numbers with $0<m_{0} \leq m_{i} \leq m_{0}^{\prime}$;

iii) for $k=0, \ldots, 1+m_{i}$

$$
\begin{array}{ll}
\left\|D^{k} Z_{2 i}(x)\right\| \leq c_{k}^{\prime}\left|x_{i}\right|^{2-k+m_{i}} & \text { if } x \in B(0,1) ; \\
\left\|D^{k} Z_{2 i}(x)\right\| \leq c_{k}^{\prime \prime}\left|x_{i}\right|^{1-k+m_{i}} & \text { if } x \in \mathbb{R}^{n} \backslash B(0,1) ;
\end{array}
$$


iv) for any $k \geq 2+m_{i}$

$$
\left\|D^{k} Z_{2 i}(x)\right\| \leq c_{k}
$$

v)

$$
\begin{aligned}
& a_{0}=a^{\prime}+c_{0}, \quad a_{1}=a^{\prime}\left(1+m_{0}\right)+c_{1}, \\
& b_{0}=b^{\prime}-c_{0}>0, \quad b_{1}=b^{\prime}\left(1+m_{0}\right)-c_{1}>a_{0} m_{0}^{\prime}
\end{aligned}
$$

with $c_{k}=\max \left\{c_{k}^{\prime}, c_{k}^{\prime \prime}\right\}$.

Remark 1. If $x \in B(0,1)$ then $\left\|D^{k} Z_{2 i}(x)\right\| \leq c_{k}^{\prime}\left|x_{i}\right|^{2-k+m_{i}} \leq c_{k}^{\prime}\left|x_{i}\right|^{1-k+m_{i}}$. Putting $c_{l}=$ $\max \left\{c_{l}^{\prime}, c_{l}^{\prime \prime}\right\}$, we deduce that for any $x \in \mathbb{R}^{n}$ have $\left\|D^{k} Z_{2 i}(x)\right\| \leq c_{k}\left|x_{i}\right|^{1-k+m_{i}}$.

\subsubsection{Global stability of the $Y_{2}$-flow on $\mathbb{R}^{n}$}

Let $\psi_{t}^{2}=\exp \left(t Y_{2}\right)$ be the $Y_{2}$-flow and let $\rho>0$ be arbitrary and fixed, so by the estimates (25) for all $x \in B(0, \rho)$ and all $t \geq t_{0}>0$ there is a constant $M_{0}>0$ such that

$$
\left\|\psi_{t}^{2}(x)\right\| \leq M_{0}\|x\| t^{-\frac{1}{m_{0}^{\prime}}}
$$

So by Proposition 1, the origin 0 is a G.A.S. equilibrium for the $Y_{2}$-flow $\psi_{t}^{2}$ on $\mathbb{R}^{n}$.

\subsubsection{Global stability of prolongation of the $Y_{2}$-flow on $\mathbb{R}^{n}$}

We proceed by recurrence. Since it is already true for $k=0$, we suppose that for any $l=$ $1, \ldots, k-1$, with $k \geq 2$, the origin 0 is a $G . A . S$. to $D^{l} \psi_{t}^{2}(x)$ on $\mathbb{R}^{n}$ that is to say for any fixed $\rho>0$, all $x \in B(0, \rho)$ and all $t \geq t_{0}>0$ there are constants $M_{l}>0$ such that

$$
\left\|D^{l} \psi_{t}^{2}(x)\right\| \leq M_{l} t^{-\frac{b_{1}}{a_{0} m_{0}^{\prime}}} \quad \text { and } \quad\left\|D^{l} \psi_{0}^{2}(x)\right\| \leq M_{l}^{\prime} .
$$

We will show that 0 is a $G$.A.S. for $D^{k} \psi_{t}^{2}(x)$ on $\mathbb{R}^{n}$.

Put $\eta_{2}^{k}(t, x, \nu, \ldots, \nu)=D^{k} \psi_{t}^{2}(x) \nu^{k} \forall \nu \in \mathbb{R}^{n}$ which is solution of the dynamic system

$$
\frac{d}{d t} \eta_{2}^{k}=D_{y} Y_{2} \cdot \eta_{2}^{k}+G_{2}^{k}(t, x, \nu), \quad \eta_{2}^{k}(0, x, \nu, \ldots, \nu)=\nu
$$

with $y=\psi_{t}^{2}(x)$ and

$$
G_{2}^{k}(t, x, \nu)=\sum_{l=2}^{k} D_{y}^{l} Y_{2}(y) \sum_{\substack{i_{1}+\cdots+i_{l}=k \\ i_{j}>0}}\left(\prod_{j=1}^{l} D^{i_{j}} \psi_{t}^{2}(x) \nu^{i_{j}}\right) .
$$

By the method of the resolvent, we deduce

$$
\eta_{2}^{k}(t, x, \nu, \ldots, \nu)=D \psi_{t}^{2}(x) \nu+\int_{0}^{t} D \psi_{t-s}^{2}\left(\psi_{s}^{2}(x)\right) G_{2}^{k}(s, x, \nu) d s .
$$

Clearly the integral

$$
I_{k}^{1}=\int_{0}^{1}\left\|D \psi_{t-s}^{2}\left(\psi_{s}^{2}(x)\right)\right\|\left\|G_{2}^{k}(s, x, \nu)\right\| d s
$$


is well defined at $s=0$ and $s=t$, since

$$
\lim _{s \rightarrow 0^{+}} D \psi_{t-s}^{2}\left(\psi_{s}^{2}(x)\right)=D \psi_{t}^{2}(x) .
$$

By the recurrent assumption $D^{l} \psi_{0}^{2}(x)$ are bounded and there exist constants $A_{l}>0$ such that

$$
\lim _{s \rightarrow 0^{+}}\left\|G_{2}^{k}(s, x, \nu)\right\| \leq \sum_{l=2}^{k} A_{l}\left\|D_{x}^{l} Y_{2}(x) \nu^{l}\right\| .
$$

In the same way

$$
\lim _{s \rightarrow t^{-}} D \psi_{t-s}^{2}\left(\psi_{s}^{2}(x)\right)=\text { identity }
$$

Now, we have to show that

$$
I_{k}^{2}=\int_{1}^{t}\left\|D \psi_{t-s}^{2}\left(\psi_{s}^{2}(x)\right)\right\|\left\|G_{2}^{k}(s, x, \nu)\right\| d s
$$

converges uniformly on any compact set $K \subset \mathbb{R}^{n}$ as $t \rightarrow 0$.

Let $x \in K$, by the relations (26) and (28) we get for all $t \geq 0$

$$
\begin{aligned}
& \|x\|\left(1+a_{0} m_{0} t\|x\|^{m_{0}}\right)^{\frac{-1}{m_{0}}} \leq\left\|\psi_{t}^{2}(x)\right\| \leq\|x\|\left(1+b_{0} m_{0}^{\prime} t\|x\|^{m_{0}^{\prime}}\right)^{\frac{-1}{m_{0}^{\prime}}}, \\
& \left(1+b_{0} m_{0} t\|x\|^{m_{0}}\right)^{-\frac{a_{1}}{b_{0} m_{0}}} \leq\left\|D \psi_{t}^{2}(x)\right\| \leq\left(1+a_{0} m_{0}^{\prime} t\|x\|^{m_{0}^{\prime}}\right)^{-\frac{b_{1}}{a_{0} m_{0}^{\prime}}} .
\end{aligned}
$$

So $\|y\|=\left\|\psi_{t}^{2}(x)\right\| \leq\|x\|$ and $\left\|D \psi_{t-s}^{2}\left(\psi_{s}^{2}(x)\right)\right\|$ is bounded. Since for any $x \in \mathbb{R}^{n}$ and any $l=1, \ldots, 1+m_{i},\left\|D^{l} Z_{2 i}(x)\right\| \leq c_{l}\left|x_{i}\right|^{1-l+m_{i}}$ then $D_{y}^{l} Y_{2}(y)$ are bounded. Now by the assumption of recurrence there exist constants $M_{l}>0$ such that for any $t>0$

$$
\left\|D^{l} \psi_{t}^{2}(x)\right\| \leq M_{l} t^{-\frac{b_{1}}{a_{0} m_{0}^{\prime}}}
$$

with $a_{0} m_{0}^{\prime}<b_{1}$ i.e. $\frac{b_{1}}{a_{0} m_{0}^{\prime}}>1$, and we deduce the existence of constants $C_{l}>0$ such that

$$
\lim _{t \rightarrow+\infty} I_{k}^{2} \leq \sum_{l=2}^{k} C_{l} \int_{1}^{+\infty} s^{-\frac{l b_{1}}{a_{0} m_{0}^{\prime}}} d s \leq \sum_{l=2}^{k} C_{l}\left(\frac{l b_{1}}{a_{0} m_{0}^{\prime}}-1\right)^{-1} .
$$

The integral $I_{k}^{2}$ converges uniformly on any compact $K \subset \mathbb{R}^{n}$ as $t \rightarrow+\infty$.

Now since the integral is well defined at $s=0$, then

$$
\lim _{t \rightarrow 0}\left\|\eta_{2}^{k}(t, x, \nu, \ldots, \nu)\right\| \leq \lim _{t \rightarrow 0}\left\|D \psi_{t}^{2}(x) \nu\right\|=\|\nu\|
$$

hence there is a constant $M_{k}^{\prime}>0$ such that

$$
\left\|D^{k} \psi_{0}^{2}(x)\right\| \leq M_{k}^{\prime}
$$

In the same way as above the integral $\int_{0}^{t}\left\|D \psi_{t-s}^{2}\left(\psi_{s}^{2}(x)\right)\right\|\left\|G_{2}^{k}(s, x, \nu)\right\| d s$ is well defined and putting $\tau=\frac{s}{t}$ we obtain

$$
\eta_{2}^{k}(t, x, \nu, \ldots, \nu)=D \psi_{t}^{2}(x) \nu+t \int_{0}^{1} D \psi_{t(1-\tau)}^{2}\left(\psi_{t \tau}^{2}(x)\right) G_{2}^{k}(t \tau, x, \nu) d \tau .
$$


Since $b_{1}=b^{\prime}\left(1+m_{0}\right)-c_{1}>a_{0} m_{0}^{\prime}$, by the estimates (26) and (28), we deduce the existence of a constant $M_{k}>0$ such that

$$
\begin{aligned}
\left\|\eta_{2}^{k}(t, x, \nu, \ldots, \nu)\right\| & \leq\left\|D \psi_{t}^{2}(x) \nu\right\|+t \int_{0}^{1}\left\|G_{2}^{k}(t \tau, x, \nu)\right\| d \tau \\
& \leq\left\|D \psi_{t}^{2}(x) \nu\right\|+t \sum_{l=2}^{k} \int_{0}^{1} \frac{(t \tau)^{-\frac{l b_{1}}{a_{0} m_{0}^{\prime}}}\|x\|^{1+m_{0}^{\prime}-l}}{\left(1+b_{0} m_{0}^{\prime} t \tau\|x\|^{m_{0}^{\prime}}\right)^{\frac{1+m_{0}^{\prime}-l}{m_{0}^{\prime}}}} d \tau \leq M_{k} t^{-\frac{b_{1}}{a_{0} m_{0}^{\prime}}}
\end{aligned}
$$

Which shows that the origin 0 is a G.A.S. equilibrium for $\eta_{2}^{k}$ on $\mathbb{R}^{n}$. We formulate this fact as

Proposition 5. Let $k \geq 0$ be any integer. Under the above conditions (i), (ii), (iii), (iv) and $(v)$, the origin 0 is a G.A.S. of order $k$ on $\mathbb{R}^{n}$ for the $Y_{2}$-flow and there is a constant $M_{k}>0$ such that for any $t \geq t_{0}>0$

$$
\left\|D^{k} \psi_{t}^{2}(x)\right\| \leq M_{k} t^{-\frac{b_{1}}{a_{0} m_{0}^{\prime}}}
$$

\subsection{Global stability of prolongations of the $Y_{3}$-flow}

Let

$$
Y_{3}=\sum_{i=1}^{n}\left(\alpha_{i} x_{i}+\beta_{i} x_{i}^{1+m_{i}}+Z_{3 i}(x)\right) \frac{\partial}{\partial x_{i}}
$$

with

i) all the coefficient $\alpha_{i}$ are negative with $-a \leq \alpha_{i} \leq-b$;

ii) all the coefficients $\beta_{i} \leq 0$ and $-a^{\prime} \leq \beta_{i} \leq-b^{\prime}$;

iii) the exponents $m_{i}$ are even natural numbers with $0<m_{0} \leq m_{i} \leq m_{0}^{\prime}$;

iv) For any $k=0, \ldots, 1+m_{i}$

$$
\begin{array}{ll}
\left\|D^{k} Z_{3 i}(x)\right\| \leq c_{k}^{\prime}\left|x_{i}\right|^{2-k+m_{i}} & \text { if } x \in B(0,1), \\
\left\|D^{k} Z_{3 i}(x)\right\| \leq c_{k}^{\prime \prime}\left|x_{i}\right|^{1-k+m_{i}} & \text { if } x \in \mathbb{R}^{n} \backslash B(0,1) ;
\end{array}
$$

v) for any $k \geq 2+m_{i}$

$$
\left\|D^{k} Z_{3 i}(x)\right\| \leq c_{k}
$$

vi)

$$
\begin{aligned}
& a_{0}=a^{\prime}+c_{0}, \quad a_{1}=a^{\prime}\left(1+m_{0}\right)+c_{1}, \\
& b_{0}=b^{\prime}-c_{0}>0, \quad b_{1}=b^{\prime}\left(1+m_{0}\right)-c_{1}>0
\end{aligned}
$$

with $c_{k}=\max \left\{c_{k}^{\prime}, c_{k}^{\prime \prime}\right\}$

Remark 2. If $x \in B(0,1)$ then $\left\|D^{k} Z_{3 i}(x)\right\| \leq c_{k}^{\prime}\left|x_{i}\right|^{2-k+m_{i}} \leq c_{k}^{\prime}\left|x_{i}\right|^{1-k+m_{i}}$. Let $c_{l}=\max \left\{c_{l}^{\prime}, c_{l}^{\prime \prime}\right\}$, for any $x \in \mathbb{R}^{n}$ one has $\left\|D^{k} Z_{3 i}(x)\right\| \leq c_{k}\left|x_{i}\right|^{1-k+m_{i}}$. 


\subsubsection{Global stability of the $Y_{3}$-flow $\mathbb{R}^{n}$}

Denote by $\psi_{t}^{3}=\exp \left(t Y_{3}\right)$, by the estimates (30), we have

$$
\left\|\psi_{t}^{3}(x)\right\| \leq C\|x\| e^{-b t} \quad \forall t>0 \text { and } \forall x \in \mathbb{R}^{n},
$$

where $C>0$ is a constant. So by Proposition 1,0 is a $G . A . S$. on $\mathbb{R}^{n}$. We proceed by recurrence; since the property is true in case $k=0$, we assume that the property remains true for any $l=1, \ldots, k-1$, with $k$ fixed i.e. 0 is a global G.A.S. of $\eta_{3}^{l}(t, x, \nu, \ldots \nu)=\left\|D^{l} \psi_{t}^{3}(x) \nu^{k}\right\|$ on $\mathbb{R}^{n}$ and there exist constants $M_{l}>0$ such that for any $t>0$

$$
\left\|D^{l} \psi_{t}^{3}(x)\right\| \leq M_{l} e^{-b t} .
$$

We will show that 0 is a G.A.S. equilibrium to $\eta_{3}^{k}$ on $\mathbb{R}^{n}$.

$\eta_{3}^{k}(t, x, \nu, \ldots, \nu)$ is a solution to the dynamic system

$$
\frac{d}{d t} \eta_{3}^{k}=D_{y} \eta_{3}^{k}+G_{3}^{k}(t, x, \nu)
$$

with $y=\psi_{t}^{3}(x)$ and

$$
G_{3}^{k}(t, x, \nu)=\sum_{l=2}^{k} D_{y}^{l} Y_{3}(y) \sum_{\substack{i_{1}+\cdots+i_{l}=k \\ i_{j}>0}}\left(\prod_{j=1}^{l} D^{i_{j}} \psi_{t}^{3}(x) \nu^{i_{j}}\right) .
$$

By the method of the resolvent, we get

$$
\eta_{3}^{k}(t, x, \nu, \ldots, \nu)=D \psi_{t}^{3}(x) \nu+\int_{0}^{t} D \psi_{t-s}^{3}\left(\psi_{s}^{3}(x)\right) G_{3}^{k}(s, x, \nu) d s
$$

and by the same argument as for the $Y^{1}$-flow, we deduce that for any integer $k \geq 0$ there exist a constant $M_{k}$ such that $\forall t \geq 0$

$$
\left\|D^{k} \psi_{t}^{3}(x)\right\| \leq M_{k}\|x\| e^{-b t} .
$$

By Proposition 1, we have

Proposition 6. Under the above conditions $(i),(i i),(i i i),(i v),(v)$ and $(v i)$, the origin 0 is a G.A.S. equilibrium of order $k$ on $\mathbb{R}^{n}$ to the $Y_{3}$-flow .

\section{References}

[1] Benalili M., Lansari A., Ideal of finite codimension in contact Lie algebra, J. Lie Theory 11 (2001), $129-134$.

[2] Benalili M., Lansari A., Une propriété des idéaux de codimension finie en algèbre de Lie des champs de vecteurs, J. Lie Theory 15 (2005), 13-26.

[3] Fenichel N., Asymptotic stability with rate conditions, Indiana Univ. Math. J. 23 (1973/1974), 1109-1137.

[4] Gutierrez C., A solution to the bidimensional global asymptotic stability conjecture, Ann. Inst. H. Poincaré Anal. Non Linéaire 12 (1995), 627-671.

[5] Hahn W., Stability of motions, Springer-Verlag, New York, 1967.

[6] Libre J., Teixeria M., Global asymptotic stability for a class of discontinuous vector fields in $\mathbb{R}^{2}$, Dyn. Syst. 122 (2007), 133-146.

[7] Shafer D.S., Structure and stability of gradient polynomial vector fields, J. London Math. Soc. (2) 41 (1990), 109-121.

[8] Meisters G.H., Global stability of dynamical systems, SIAM Rev. 31 (1989), 147-151.

[9] Zajtz A., Global version of a Sternberg linearization theorem, Roczik Nauk. Dydact. Prace Mat. (2000), no. 17, 265-269.

[10] Zajtz A., Some division theorems for vector fields, Ann. Polon. Math. 58 (1993), 19-28. 